immunostimulatory activity of the PD-1 inhibitor pembrolizumab may be enhanced by concurrent chemoradiotherapy (CCRT). After the KEYNOTE-158 study, in which pembrolizumab showed durable antitumor activity, pembrolizumab monotherapy was approved for patients with PD-L1-positive recurrent or metastatic cervical cancer who progressed during or after chemotherapy. ENGOT-cx11/GOG 3047/KEYNOTEA18 (NCT04221945) is a phase 3, randomized, placebo-controlled study evaluating pembrolizumab with CCRT for the treatment of high-risk, locally advanced cervical cancer.

Methodology Approximately 980 patients with high-risk (International Federation of Gynecology and Obstetrics 2014 stage IB2-IIB with node-positive disease or stage IIIIVA), locally advanced, histologically confirmed cervical cancer who have not received systemic therapy, immunotherapy, definitive surgery, or radiation will be randomized 1:1 to receive either 5 cycles of pembrolizumab $200 \mathrm{mg}$ every 3 weeks $(\mathrm{Q} 3 \mathrm{~W})$ + CCRT followed by 15 cycles of pembrolizumab $400 \mathrm{mg}$ Q6W or 5 cycles of placebo Q3W + CCRT followed by 15 cycles of placebo Q6W. CCRT includes 5 cycles (optional 6th dose) of cisplatin $40 \mathrm{mg} / \mathrm{m}^{2} \mathrm{Q} 1 \mathrm{~W}+$ EBRT followed by brachytherapy. Randomization is stratified by planned EBRT type (intensity-modulated radiotherapy [IMRT] or volumetric-modulated arc therapy [VMAT] vs non-IMRT or non-VMAT), cancer stage at screening (stage IB2-IIB vs III-IVA), and planned total radiotherapy dose. Treatment will continue until the patient has received 20 cycles of pembrolizumab (5 cycles $200 \mathrm{mg}$ Q3W, 15 cycles $400 \mathrm{mg}$ Q6W) vs placebo ( 2 years) or until disease progression, unacceptable toxicity, or withdrawal. Primary endpoints are progression-free survival (PFS) per Response Evaluation Criteria in Solid Tumors version 1.1 by investigator and overall survival (OS). Secondary endpoints include PFS by blinded independent central review, PFS at 2 years, OS at 3 years, complete response at 12 weeks, objective response rate, PFS and OS by PD-L1 status, quality of life, and safety. Enrolment began May 2020 and is planned for 193 sites in 30 countries.

Klikně te nebo klepně te sem a zadejte text.

\section{MRI BASED TUMOUR RADIOMICS IN CARCINOMA CERVIX: A POTENTIAL PREDICTOR OF RESPONSE TO CHEMORADIATION}

'S Chopra, ${ }^{2} \mathrm{P}$ Nayak, 'Y Gupta, 'I Swamidas, 'IS Goda, 'S Panda, 'L Gurram, '3 Rath, ${ }^{4} \mathrm{P}$ Popat, ${ }^{2} \mathrm{U}$ Mahantshetty, ${ }^{3} \mathrm{~S}$ Gupta, ${ }^{2} \mathrm{JP}$ Agarwal. 'ACTREC, Tata Memorial Centre, Radiation Oncology, Navi Mumbai, India; ${ }^{2}$ Tata Memorial Hospital, Tata Memorial Centre, Radiation Oncology, Mumbai, India; ${ }^{3} T a t a$ Memorial Hospital, Tata Memorial Centre, Medical Oncology, India; ${ }^{4}$ Tata Memorial Hospital, Tata Memorial Centre, Radiology, India

\subsection{6/ijgc-2021-ESGO.22}

Introduction/Background* To determine if baseline T2weighted (T2W) MRI texture parameters can predict response to chemoradiation in cervical carcinoma.

Methodology Seventy-four patients of locally advanced carcinoma cervix treated with definitive chemoradiation (45Gy in 25 fractions and weekly cisplatin $\left(40 \mathrm{mg} / \mathrm{m}^{2}\right)$ and image guided brachytherapy between 2017 and 2019 were included. Gross tumour volume (GTV) and high risk clinical target volume (HRCTV) was delineated on T2W MRI at baseline and at brachytherapy using the texture analysis software. Tumour regression $>75 \%$ was considered as a surrogate of good response. Multiple tumour slices were sampled and first order

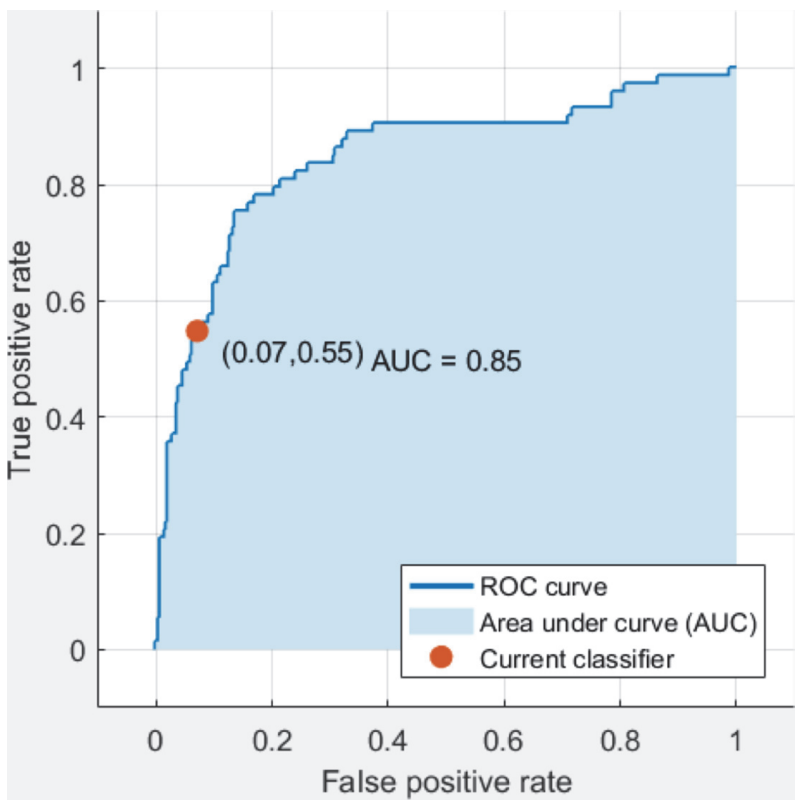

\section{Abstract 262 Figure 1}

statistics were applied to produce 6 texture parameters, namely mean intensity, standard deviation, entropy, mean positive pixels, skewness, and kurtosis. After using fine,moderate, and coarse anatomical filters a total of 36 texture variables were generated.

Clinical variables namely histological subtype, tumour grade, FIGO 2009 stage and nodal status were documented. These clinical variables along with texture parameters were compared with treatment response using the Mann-Whitney U test. Lasso regression was used to select texture parameters that best correlated with treatment response. These were used to develop support vector machine (SVM) models which were validated using 10-fold cross validation. The most parsimonious model was described in terms of area under curve (AUC) and metrics of diagnostic accuracy.

Result(s)* The median age was 50 years (range 34 to 65). Overall, $63(85 \%)$ had squamous cell carcinoma. Half of the included patients $(37 / 74)$ had positive pelvic or para-aortic lymph nodes. As per FIGO 2018 criteria 23 (31\%) and 51 (69\%) patients were stage II and III, respectively. Good response was seen in $10 / 74$ patients. None of the clinical variables discriminated between response. However, high mean and skewness, and low entropy and kurtosis did significantly correlate with poor response. Using 15 selected features, the best SVM model had an AUC of 0.85 and accurately classified $86.5 \%$ cases, with sensitivity and specificity of $55 \%$ and $93 \%$, respectively.

Conclusion* Tumour radiomics can be utilized to predict response to chemoradiation whereas baseline clinical parameters do not predict for response. Baseline texture analysis can be an important tool to predict response to chemoradiation.

\section{SOCIOECONOMIC FACTORS ASSOCIATED WITH PREVALENCE AND STAGING OF CERVICAL CANCER}

MG Theresa*, FW Wagey, BJ Laihad, FM Wagey. Manado, Obstetric and Gynecology Prof. Dr. R. D. Kandou General Hospital, Manado, Manado, Indonesia

10.1136/ijgc-2021-ESGO.23 
Introduction/Background* Cervical cancer ranks fourteenth among all types of cancer. Several factors of socioeconomic status, including education level, and individual or environmental economic status, have been associated with the risk of advanced cancer at diagnosis.

Methodology This cross-sectional study was conducted on 108 female diagnosed with cervical cancer at the Obstetrics and Gynecology Department of Prof. Dr. RD Kandou Man-

Abstract 284 Table 1 Distribution of demographic characteristics of studied women

\begin{tabular}{|c|c|c|c|}
\hline Characteristics & Total & Early stage & Late stage \\
\hline \multicolumn{4}{|l|}{ Age at diagnosis } \\
\hline $25-39$ & 44 & 28 & 16 \\
\hline $40-59$ & 61 & 36 & 25 \\
\hline$>60$ & 3 & 1 & 2 \\
\hline Mean \pm SD & $36 \pm 24.3$ & $21.6 \pm 14.9$ & $14.3 \pm 9.4$ \\
\hline \multicolumn{4}{|l|}{ Pap Smear screening } \\
\hline Ever & 27 & 21 & 6 \\
\hline Never & 81 & 45 & 37 \\
\hline \multicolumn{4}{|l|}{ Active sexual intercourse } \\
\hline Yes & 64 & 45 & 19 \\
\hline Not & 44 & 20 & 24 \\
\hline \multicolumn{4}{|l|}{ Sexual partner } \\
\hline$>1$ & 19 & 3 & 16 \\
\hline 1 & 89 & 62 & 27 \\
\hline \multicolumn{4}{|l|}{ Age at first sexual intercourse } \\
\hline$\leq 20$ years & 11 & 4 & 7 \\
\hline$>20$ years & 97 & 61 & 36 \\
\hline \multicolumn{4}{|l|}{ Education level } \\
\hline No school & 5 & 1 & 4 \\
\hline Primary & 7 & 3 & 4 \\
\hline Junior High School & 19 & 8 & 11 \\
\hline Senior High school & 23 & 11 & 12 \\
\hline Diploma & 15 & 9 & 6 \\
\hline College & 39 & 33 & 6 \\
\hline Mean \pm SD & $18 \pm 11.3$ & $10.6 \pm 10.4$ & $7.2 \pm 3.2$ \\
\hline \multicolumn{4}{|l|}{ Job } \\
\hline Civil servants & 35 & 24 & 11 \\
\hline Private & 23 & 15 & 8 \\
\hline Traders & 16 & 9 & 7 \\
\hline Farmer & 8 & 3 & 5 \\
\hline Housewife/not working & 26 & 14 & 12 \\
\hline Mean \pm SD & $21.6 \pm 9.1$ & $13 \pm 6.9$ & $8.6 \pm 2.6$ \\
\hline \multicolumn{4}{|l|}{ Income } \\
\hline$<$ Rp. $1,500,000$ & 31 & 13 & 18 \\
\hline Rp. $1,500,000$ - Rp. $2,500,000$ & 14 & 5 & 9 \\
\hline Rp. $2,500,000-3,500,000$ & 35 & 22 & 13 \\
\hline$>$ Rp. $3,500,000$ & 28 & 25 & 3 \\
\hline Mean \pm SD & $27 \pm 7.9$ & $16.3 \pm 7.8$ & $10.75 \pm 5.5$ \\
\hline
\end{tabular}

ado. A researcher-made questionnaire was used to collect data about social economic status. The outcomes were analyzed using linear regression methods. Data were analyzed using SPSS 23.

Result(s)* The mean age \pm standard deviation (SD) of subjects in this study was $36 \pm 24.3$ where the early stage was 21.6 \pm 14.9 with 36 subject $(n=65)$ were in age $40-58$ year old and the late stage was $14.3 \pm 9.4$ with 25 subject $(n=43)$ were in age 40-59 year old. Results of linear regression test showed that there is a significant statistical relationship between early stage and high education level $(\mathrm{P}<0.001)$ better job $(\mathrm{P}<0.001)$ and high income $(\mathrm{P}<0.002)$.

Conclusion* There is significant relationship between socioeconomic status and prevalence of cervical cancer. A better job can leads to a better income and also high education level so that woman can access the healthcare facility to prevent the disease with consistent screening or to treat the cancer on the very early stage.

\section{INDOCYANINE GREEN TO ASSESS VASCULARITY OF ILEAL CONDUIT ANASTOMOSIS DURING PELVIC EXENTERATION FOR RECURRENT/PERSISTENT GYNECOLOGICAL CANCER}

${ }^{1} \mathrm{~N}$ Bizzarrit, ${ }^{2} \mathrm{~N}$ Foschi, ${ }^{1} \mathrm{M}$ Loverro, ${ }^{1} \mathrm{~L}$ Tortorella, ${ }^{3} \mathrm{~F}$ Santullo, ${ }^{1} \mathrm{~A}$ Rosati, ${ }^{1} \mathrm{~S}$ Gueli Alletti, ${ }^{1} \mathrm{~B}$ Costantini, ' $\mathrm{V}$ Gallotta, ${ }^{1} \mathrm{MG}$ Ferrandina, ${ }^{1} \mathrm{~A}$ Fagotti, ${ }^{1} \mathrm{~F}$ Fanfani, ${ }^{1} \mathrm{G}$ Scambia, ${ }^{1,4} \mathrm{G}$ Vizzielli. ${ }^{1}$ Fondazione Policlinico Universitario A. Gemelli, IRCCS, UOC Ginecologia Oncologica, Dipartimento per la salute della Donna e del Bambino e della Salute Pubblica, Rome, Italy; ${ }^{2}$ Fondazione Policlinico Universitario A. Gemelli, IRCCS, , UOC Clinica Urologica, Rome, Italy; ${ }^{3}$ Fondazione Policlinico Universitario A. Gemelli, IRCCS, UOC Chirurgia Peritoneo e Retroperitoneo, Dipartimento Scienze mediche e chirurgiche "Italy; ${ }^{4}$ University of Udine, Obstetrics and Gynecology Department, Udine, Italy

\subsection{6/ijgc-2021-ESG0.24}

Indocyanine green to assess vascularity of ileal conduit anastomosis during pelvic exenteration for recurrent/persistent gynecological cancer: a pilot study.

Introduction/Background* Pelvic exenteration performed for recurrent/persistent gynecological malignancies has been associated to urological short- and long-term morbidity, due to altered vascularization of tissues for previous radiotherapy. The aims of the present study were to describe the use of intravenous indocyanine green (ICG) to assess vascularity of urinary diversion (UD) after pelvic exenteration for gynecologic cancers, to evaluate the feasibility and safety of this technique and to assess the post-operative complications.

Methodology Prospective, observational, single-center, pilot study including consecutive patients undergoing anterior or total pelvic exenteration due to persistent/recurrent gynecologic cancers between $08 / 2020$ and $03 / 2021$ at Fondazione Policlinico Gemelli IRCCS, Rome, Italy. All patients underwent intravenous injection of $3-6 \mathrm{ml}$ of ICG $(1.25 \mathrm{mg} / \mathrm{ml})$ once the

Abstract 284 Table 2 Results of linear regression

\begin{tabular}{|c|c|c|c|c|c|c|}
\hline \multirow[t]{2}{*}{ Independent Variable } & \multicolumn{3}{|l|}{ Early stage } & \multicolumn{3}{|l|}{ Late stage } \\
\hline & The Regression Coefficient & Confidence Level & $P$ Value & The Regression Coefficient & Confidence Level & $P$ Value \\
\hline High Education level & 3.9 & $2.03-5.8$ & 0.001 & 0.4 & $-2.1-2.01$ & 0.97 \\
\hline Better job & 2.7 & $1.1-4.3$ & 0.001 & 3.1 & $-2.4-8.5$ & 0.3 \\
\hline High income & -5.2 & $-8.4-1.9$ & 0.002 & 2.7 & $-5.5-10.9$ & 0.5 \\
\hline
\end{tabular}

